7. A. Lebow and M. Schechter, Semigroups of operators and measures of noncompactness, J. Functional Analysis 7 (1971), 1-26. MR 42 \#8301.

8. E.-O. Liebetrau, Über die -Fredholmmenge linearer Operatoren, Dissertation, Dortmund, 1972.

9. G. Pólya and G. Szegö, Problems and theorems in analysis. Vol. 1, Die Grundlehren der math. Wissenschaften, Band 193, Springer-Verlag, Berlin and New York, 1972.

FACHBERICH MATHEMATIK, UNIVERSITÄT OLDENBURG, OLDENBURG, GERMANY (Current address of K.-H. Förster)

WISKUNDIG SEMINARIUM, VRIJE UNIVERSITEIT, AMSTERDAM, THE NETHERLANDS

Current address (M. A. Kaashoek): Department of Mathematics, University of Maryland, College Park, Maryland 20742

PROCEEDINGS OF THE

AMERICAN MATHEMATICAL SOCIETY

Volume 49. Number 1, May 1975

\title{
CHARACTERIZATIONS OF THE COMPLEX PROJECTIVE PLANE BY CURVATURE
}

\author{
ROBERT E. GREENE AND HARSH PITTIE
}

ABSTRACT. We give short proofs to show that under various positivity assumptions on the curvature of a Kähler surface $X$, it is biholomorphically equivalent to $P_{2}(C)$. In particular, the case of $\delta$-holomorphic pinching $>1 / 2$ (Theorem 1 ) is best possible and, we believe, new.

Let $X$ be a compact Kähler manifold of complex dimension 2. Given a real two-dimensional subspace $p$ of the real tangent space of $X$ at some point, we denote by $K(p)$ the Riemannian curvature of $p . X$ is called $\delta$-bomomorpbically pinched if there is some constant $A>0$ such that $\delta A \leq K(p) \leq A$ for all holomorphic planes $p$, i.e. for all planes $p$ invariant under the complex structure endomorphism $J$. Given two holomorphic planes

Received by the editors November 13, 1973 and, in revised form, February 11, 1974.

AMS (MOS) subject clas sifications (1970). Primary 32C10, 32J15, 53C20.

Key words and phrases. Kähler manifold, curvature, pinching, Chern class, Pontrjagin class, Riemann-Roch, Kodaira vanishing the orem. 
$p$ and $p^{\prime}$, the holomorphic bisectional curvature $H\left(p, p^{\prime}\right)$ associated to $p$ and $p^{\prime}$ is defined to be $R(X, J X, Y, J Y)$ where $X$ and $Y$ are unit vectors in $p$ and $p^{\prime}$, respectively, and $R$ is the Riemannian curvature tensor. For further details see Kobayashi and Nomizu [11].

Theorem 1. If $X$ is $\delta$-homomorphically pinched, $\delta>1 / 2$, then $X$ is bibolomorphically bomeomorphic to $P_{2}(C)$.

Theorem 2 (Andreotti-Frankel). If $X$ has positive sectional curvature, i.e. $K(p)>0$ for all $p$, then $X$ is bibolomorpbically bomeomorphic to $P_{2}(C)$.

Theorem 2' (Goldberg-Kobayashi). If $X$ bas positive bolomorpbic bisectional curvature, then $X$ is bibolomorphically homeomorphic to $P_{2}(C)$.

Remarks. In Theorem 1 , the number $1 / 2$ cannot be reduced, since the metric product $P_{1}(C) \times P_{1}(C)$ with the Fubini-Study metric on each factor has holomorphic pinching $1 / 2$. Theorem 2 is due to Andreotti and Frankel (Frankel [4]); the ir proof and the proof by Goldberg and Kobayashi of the closely related Theorem $2^{\circ}$ (Goldberg and Kobayashi [5]) as well depend upon the classification of algebraic surfaces. Our proofs are quite short and are independent of the classification theory.

Proof of Theorems 1, 2 and $2^{\prime}$. Since the proofs of the three theorems are very similar, we give the proofs together. Suppose that $X$ satisfies the hypothesis of one of the Theorems 1,2 , or $2^{\prime}$. Then:

(a) The Ricci tensor of $X$ is positive definite. This fact follows immediately under the hypothesis of positivity of sectional curvature and follows from curvature computations given in Berger [1] and GoldbergKobayashi [5] under the hypotheses of $\delta$-holomorphic pinching, $\delta>1 / 2$, and positivity of holomorphic bisectional curvature, respectively.

(b) $H^{2}(X, R)=R$. This result, which is proved using a technique of Bochner and Lichnerowicz, is proved under the hypothesis of $\delta$-holomorphic pinching, $\delta>1 / 2$, by Bishop and Goldberg [2] and under the hypothesis of positivity of holomorphic bisectional curvature by Goldberg and Kobayashi [5].

(c) $X$ is simply connected. A theorem of Kobayashi (Kobayashi [10]) states that any compact Kähler manifold with positive definite Ricci tensor is simply connected; thus (c) follows from (a).

(d) The first Chern class $c_{1}(X)$ is a positive multiple of the Kähler class of $X$. This statement follows from (a) and (b) and the standard formula 
expressing the Chern classes in terms of the curvature form (Chern [3]).

Using the se facts we can compute the first Chern and Pontrjagin classes of $X$. Since $X$ is simply-connected, $H^{2}(X: Z)$ is free, by the universal coefficient theorem; since the second Betti number of $X$ is $1, H^{2}(X: Z)$ is infinite cyclic. Choose $\xi$ to be the generator which is a positive multiple of the Kähler class $\omega$. By Poincaré duality, $\xi^{2}$ is a generator for $H^{4}(X: Z)$. In fact it is the class dual to the orientation class, i.e., $\xi^{2}[X]=1$. This is because $\xi^{2}$ is a positive multiple of $\omega \wedge \omega$, which is a positive multiple of the volume form. Thus the integral cohomology ring is easily seen to be $H^{*}(X: Z)=Z[\xi] /\left(\xi^{3}\right) .^{1}$

Therefore the signature of $X$ is 1 , and by the Hirzebruch-Thom theorem [6] the first Pontrjagin class $p_{1}(X)=3 \xi^{2}$. Now we have the relation $c_{1}(X)^{2}=2 c_{2}(X)+p_{1}(X)$. (This relation is valid quite generally for complex vector-bundles-see Hirzebruch [6, Chapter 1], or Hirzebruch and Hopf [7] for the case of 4-manifolds.) In the case at hand, $c_{2}(X)$ is the Euler class, so $c_{1}(X)^{2}=9 \xi^{2}$. Since $c_{1}(X)$ is a positive multiple of $\xi, c_{1}(X)=3 \xi$.

One can now apply the argument of Hirzebruch and Kodaira [8] as given for example in Morrow [13]. Morrow shows that if $X$ is homeomorphic to $P_{2}(C)$ and the first Chern class is positive, then $X$ is necessarily biholomorphically homeomorphic to $P_{2}(C)$. However, the assumption that $X$ is homeomorphic to $P_{2}(C)$ is actually used only to calculate the $\hat{A}$-genus of $X$ and thence the Chern class $c_{1}(X)$. Since we already know $c_{1}(X)$ and $p_{1}(X)$ we can continue the reasoning without the homeomorphism assumption: Since $\xi$ is of the type $(1,1)$, there is a holomorphic complex line bundle $E \rightarrow X$ corresponding to $\xi$. By the Riemann-Roch theorem and the Kodaira vanishing theorem, one finds that $\operatorname{dim}_{C} \Gamma(E)=\operatorname{dim}_{C} H^{0}(X, O(E))=3$ and that, for some large integer $s, E^{s}$ is ample. Then the "meromorphic" map $\phi$ : $X \rightarrow P_{2}(C)$ given by three linearly independent sections $\left\{\phi_{0}, \phi_{1}, \phi_{2}\right\}$ of $E$ can be shown by a standard argument using the Segre embedding to be a biholomorphic home omorphism (Morrow [13, pp. 319-320]; cf. Howard [9], Kobayashi and Ochiai [12]).

1 This already implies that $X$ has the homotopy type of $C P_{2}$, since $\pi_{1}(X)=0$. Howe ver, we do not need this.

\section{RE F ERENCES}

1. M. Berger, Pincement riemannien et pincement holomorphe, Ann. Scuola Norm. Sup. Pisa (3) 14 (1960), 151-159; correction, ibid. (3) 16 (1962), 297. MR 25 \#3477; 28 \#558.

2. R. L. Bishop and S. I. Goldberg, On the second cohomology group of a Kaehler 
manifold of positive curvature, Proc. Amer. Math. Soc. 16 (1965), 119-122. MR 30 \#2441.

3. S.-S. Chern, Characteristic classes of Hermitian manifolds, Ann. of Math. (2) 47 (1946), 85-121. MR 7, 470.

4. T. T. Frankel, Manifolds with positive curvature, Pacific J. Math. 11 (1961), 165-174. MR 23 \#A600.

5. S. I. Goldberg and S. Kobayashi, Holomorphic bisectional curvature, J. Differ ential Geometry I (1967), 225-233. MR 37 \#3485.

6. F. Hirzebruch, Neue topologische Methoden in der algebraischen Geometrie, Ergebnisse der Mathematik und ihrer Grenzgebiete, Heft 9, Springer-Verlag, Berlin, 1956; English transl., Die Grundlehren der math. Wissenschaften, Band 131, Springer-Verlag, Berlin; Academic Press, New York, 1966, p. 86. MR 18, 509; 34 \#2573.

7. F. Hirzebruch and H. Hopf, Felder von Flächenelementen in 4-dimensionalen Mannigfaltigheiten, Math. Ann. 136 (1958), 156-172. MR 20 \#7272.

8. F. Hirzebruch and $\mathrm{K}$. Kodaira, On the complex projective spaces, J. Matho Pures Appl. (9) 36 (1957), 201-216. MR 19, 1077.

9. A. Howard, A remark on Kählerian pinching, Tohoku Math. J. 24 (1972), 11-19.

10. S. Kobayashi, On compact Kähler manifolds with positive definite Ricci tensor, Ann. of Math. (2) 74 (1961), 570-574. MR 24 \#A2922.

11. S. Kobayashi and K. Nomizu, Foundations of differential geometry. Vol. II, Interscience Tracts in Pure and Appl. Math., no. 15, Inter science, New York, 1969, 1969, pp. 368-373. MR 38 \#6501.

12. S. Kobayashi and T. Ochiai, Characterization of complex projective spaces and hyperquadrics, J. Math. Kyoto Univ. 13 (1973), 31-47.

13. J. A. Morrow, A survey of some results on complex Kähler manifolds, Global Analysis (Papers in Honor of K. Kodaira), Univ. of Tokyo Press, Tokyo, 1969, pp. 315-324. MR $41 \# 2719$.

DEPARTMENT OF MATHEMATICS, UNIVERSITY OF CALIFORNIA AT LOS ANGELES, LOS ANGELES, CALIFORNIA 90024 (Current address of R. E. Greene)

DEPARTMENT OF MATHEMATICS, COURANT INSTITUTE, NEW YORK UNIVERSITY, NEW YORK, NEW YORK 10012

Current address (Harsh Pittie): Mathematics Institute, University of Warwick, Coventry, England 